\title{
Nurses' lived experience serving on unit-based councils: A literature review
}

\author{
Alanoud Hamad RN Al-Marri ${ }^{1}$, Vahe Kehyayan*2 \\ ${ }^{1}$ Hamad Medical Corporation, Doha, Qatar \\ ${ }^{2}$ Faculty of Nursing, University of Calgary in Qatar, Doha, Qatar
}

Received: May 3, 2018

Accepted: July 1, 2018

Online Published: July 12, 2018

DOI: $10.5430 /$ jnep.v8n12p21

URL: https://doi.org/10.5430/jnep.v8n12p21

\begin{abstract}
Introduction: Shared governance (SG) is an organizational model that allows frontline nurses to have control over their daily work environment and nursing practice. Unit-based councils (UBC) are an important operational element of SG and its members are frontline nursing staff.

Purpose and methods: The purpose of this paper is to review the literature on UBCs and SG, staff nurses' perceptions, and factors that influence their adoption and successful implementation.

Results: Five major themes emerged from the literature: perception of SG; leadership implications; improvement in patient care; increase in job satisfaction; and improvement in work environment.

Conclusions: Nurses serving on UBCs have perspectives different from managers on the success of SG and UBCs. SG is viewed as a journey that requires continuous support from nurse leaders to address any issues that may arise during this journey.
\end{abstract}

Key Words: Shared governance, Unit-based councils, Nurses' experience OR perspective OR view OR attitude, Healthcare

\section{INTRODUCTION}

In recent two decades, healthcare organizations began adopting shared governance (SG) as their organizational model. Many benefits have been attributed to SG environments from the perspective of patients, staff, and the organizations themselves. In patients, SG environments are associated with improved quality outcomes such as low mortality and comorbidity rates and low adverse events. ${ }^{[1]}$ For nurses, such environments have empowered them to have the authority, autonomy and accountability for making decisions about their work environments and nursing practice at the point of care where their expertise is recognized. ${ }^{[2-4]}$ As a result, evidence has shown decreased turnover rates in nurses, higher retention rates, and lower absenteeism rates along with decreased associated costs to the organization. ${ }^{[5-8]}$ Thus, an SG governance organizational model improves an organization's financial status by cost saving and cost reduction. ${ }^{[9]}$

In an SG organizational structural model, Unit-based Councils (UBCs) "were established to give frontline staff a voice in clinical decision-making processes at the unit, divisional, and organizational levels as best practices emanate from a unit or a division and are disseminated throughout the system". [10] (p.88) In 2014, the Hamad Medical Corporation $(\mathrm{HMC}){ }^{[11]}$ the primary healthcare provider in the State of Qatar, adopted SG as its organizational model as a requirement to achieve Magnet Designation by the American Nurse Credentialing Center. ${ }^{[12]}$ To operationalize SG at the patient care unit level, HMC established UBCs on each patient care unit in all of its 10 general and specialty hospitals. Frontline nurses working on these patient care units were elected or nominated for election to serve on these councils. However,

*Correspondence: Vahe Kehyayan; Email: vkehyaya@ucalgary.ca; Address: Faculty of Nursing, University of Calgary in Qatar, Doha, Qatar. 
the nurses serving on these councils and middle and senior nurse managers have expressed different perspectives on the success of the implementation of SG and UBCs (Personal communication, January, 2017). Staff nurses are of the view that the introduction of SG and UBCs had not made any difference in their work environment. While they attend UBC meetings, they are not discussing issues pertaining to their work environment and nursing practice issues. Further, they are confused about what was expected of them and what their exact role was serving on the councils. The senior nurse manager of the patient care units agreed with the nurses' viewpoint, but the middle nurse managers of these same units supported the model and considered it to have had a positive effect on empowering frontline nurses to make decisions about patient care and their work environment at the point of care. Therefore, the purpose of this literature review was to determine what is known on nurses' lived experience serving on UBCs. The review was guided by the following questions: What is known in the literature on UBCs, SG, and nurses' lived experience with them? Are nurses making decisions about their practice, standards of care, and their work environment? And do they feel empowered in making patient care decisions at the point of care?

\section{METHOD}

The method used for this paper was a literature review. It is defined as "An extensive, systematic, and critical review of the most important published scholarly literature on a particular topic". ${ }^{[13]}$

\section{Search strategy}

A search of the literature published from January 2006 to January 2017 was conducted using CINAHL, Academic Search Complete, and Academic Search Elite databases. The following search terms or phrases were used: "shared governance and nurses' experience OR perspective OR view OR attitude", "shared governance AND healthcare", and "unitbased councils". Only full-text papers in English published in peer-reviewed journals were selected for review.

Figure 1 outlines the literature search strategy and its outcomes. The search was conducted sequentially. An initial search of the three databases resulted in 128 articles. After removing duplicates $(n=15)$, the titles and abstracts of the remaining 113 papers were reviewed. Thirteen papers were removed based on title screening, and a further 48 were removed based on abstract review. The remaining 52 full-text articles were critically appraised using the Mixed Method Appraisal Tool (MMAT) resulting in the elimination of 35 additional papers because they lacked quality. The reference lists of the remaining 17 papers were reviewed and three papers were selected resulting in a total number of 20 relevant articles that met the inclusion criteria.

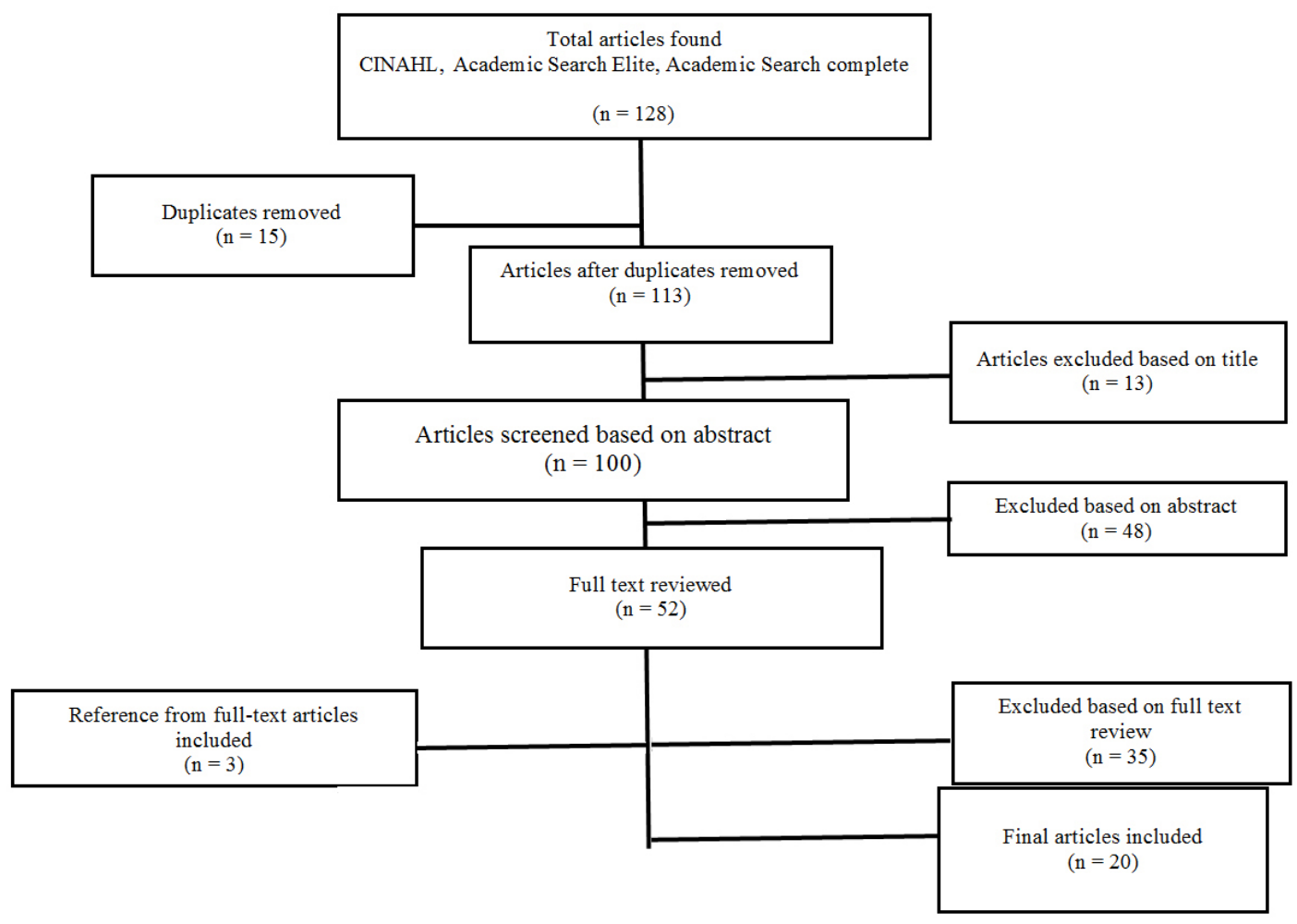

Figure 1. Literature search strategy and outcomes 
These 20 research papers using different designs explored nurses' perceptions of UBCs and SG and their impact on nurses. These studies were conducted in the following countries: Belgium, Brazil, England, Jordan, the Kingdom of Saudi Arabia (KSA), and the USA. The settings of these research studies included non-federal acute care hospitals, community medical centers, public university hospitals, not for-profit rural hospitals, academic hospitals, Magnet and non-magnet hospitals, rural hospitals, and tertiary care hospitals. There were some studies that were conducted on specialized units such as medical-surgical, cardiovascular, inpatient, outpatient, and ambulatory care.

\section{Results}

\subsection{Perception of UBCs and SG}

The literature showed diverse ways in how nurses perceived SG and UBCs. In a community medical center in Fairfield County, Connecticut, United States (USA), Gerard, Owens, and Oliver (2016) studied the level of dissonance in nurses' desired and perceived decision-making. ${ }^{[14]}$ They described dissonance as the gap between nurses' perceived and the desired level of decisional involvement (DI). The researchers reported a statistically significant difference in the levels of dissonance. They found that nurses serving on UBCs for more than five years reported significantly higher dissonance than nurses serving less than five years. They also found differences in levels of dissonance in nurses' employment status - nurses working full-time, part-time, or per diem (paid on a daily rate) with the per diem status nurses reporting the least amount of dissonance. In another study, Mangold et al. (2006) also measured decisional dissonance in registered nurses (RN) and reported statistically significant differences as well with the RNs wishing for more DI than they actually experienced. ${ }^{[15]}$ The mean score for preferred DI was low suggesting that nurses did not wish to be actively involved in institutional decision-making possibly because they were already overwhelmed with their roles and responsibilities. An alternate explanation offered by the authors was that the nurses were already satisfied in DI and hence there was no need for further involvement.

Winslow, Hougan, DeGuzman, and Black (2015) measured nurses' experience with SG and UBCs in a 176-bed community hospital in Central Virginia, USA using a survey questionnaire. ${ }^{[16]}$ Seventy seven percent (77\%) of the respondents agreed that the SG structure gave them a voice in shared decision-making. Of these, a majority (66\%) were involved in SG activities at the unit or hospital level. The majority $(80 \%)$ of the nurses had received from management adequate resources and encouragement to support the success of councils. There was a statistically significant difference between Published by Sciedu Press the perceptions of nurses serving on UBCs and nursing leadership regarding the size of UBCs and member selections. UBC members viewed the size of membership and the process of member elections as appropriate, but the leadership did not. This may be indicative that nursing leadership did not support the size of UBC membership and the process of UBC member selection. ${ }^{[16]}$ Three themes about SG were concluded from this study: it is "a pathway for nurses to have a strong voice"; it is "ownership of a best practice environment"; and it is about interdisciplinary collaboration across the hospital "identifying issues and collaborating to determine solutions" (p. 50). The authors concluded that nurses in Magnet designated hospitals had higher awareness and involvement in shared decision-making than nurses working in non-Magnet hospitals.

In a mixed methods concurrent triangulation design study in a large public university hospital in Brazil, dos Santos and Erdmann (2015) aimed to construct an interpretative model of governance in professional nursing practice. They obtained qualitative data from interviewing nurses and quantitative data from the Brazilian Nursing Work Index - Revised. The Index measures characteristics of nurses' practice environment. ${ }^{[17]}$ The qualitative study showed that "governance in nursing is based on managing nursing care and services. To perform these tasks, nurses seek to get around organizational support constraints and develop management knowledge and skills". ${ }^{[17]}$ (p. 1029) The results from the Index showed that nurses within SG organizations perceived themselves to have autonomy, control over the environment, and positive relationships with physicians.

In a Jordanian University hospital, Al-Faouri, Al Ali, and Essa (2014) examined the difference in the perception of staff nurses and nurse managers about the impact of SG. ${ }^{[18]}$ They also examined if there was a relationship between the perception of SG and nurses' demographic characteristics. They used the Hess Index of Professional Nursing Governance (IPNG) scale, which measures the perception of SG impact in six dimensions: nursing personnel, information, resources, participation, practice, and goals. The results of the study showed that there was no significant difference in the perception of SG between staff nurses and nurse managers. As well, both staff nurses and managers perceived that decisions were equally shared in all IPNG subscales. Also, there was no significant correlation between age, gender, management level, education, special training, and SG perception subscales. However, there was a statistically significant difference in the perception of SG by nurses working in different nursing units. Those who worked in critical care units and operating rooms perceived more involvement in SG activities than those working in the medical unit. Yet, nurses were not 
fully aware of the concept of SG, and they perceived having limited ability to participate on committees that related to multidisciplinary professionalism, organizational budgets, and expenses. Accordingly, the authors stressed the vital role of nursing leadership to focus and train nurses and their managers about SG and to involve nurses in decision-making. They also concluded that nursing educators need to focus more on the concept of SG and decisional involvement with nursing students. Training in SG skills should be available for all nurses, and especially for nurse managers.

In two, not for-profit hospitals in Easton and Cambridge, Maryland, USA, Wilson, Speroni, Jones, and Daniel (2014) studied the perceptions of direct care nurses and nurse managers of factors affecting nurses' participation in unit-based and general SG activities. ${ }^{[19]}$ The study as well studied factors that affected nurse engagement. The authors defined an engaged nurse as one "who's fully involved and enthusiastic about his or her work and who acts in a way that furthers nursing on the unit" (p. 21). The authors identified four factors that were rated equally 'very important' by both nurses and their managers: "direct care nurses perceiving support by unit managers; direct care nurses perceiving nurses on a unit working as team; direct care nurses feeling time to participate in activities without disrupting patient care; and direct care nurses believing they will be paid for activities beyond scheduled shift" (p. 21). However, the authors identified two significant differences in the participants' perceived factors affecting nurses' participation in SG activities: $76.6 \%$ of direct care nurses compared to $100.0 \%$ of managers reported the importance of nurses on a unit to work as a team; and $62.0 \%$ nurses compared to $93.3 \%$ managers reported that nurses felt "they had the ability to make changes at the unit level" (p. 21). Furthermore, there was a significant difference in the degree of engagement of managers (86.7\%) in in SG activities compared to direct care nurses (36.4\%).

In a cross-sectional descriptive study in an academic hospital in South Eastern Florida, USA, Lamoureux, Judkins-Cohn, Butao, McCue, and Garcia (2014) also used Hess's IPNG to test five hypotheses related to the concept of SG that there were no differences in the perception of governance between the units of the hospital, between various levels of experience, between various levels of education and certification, between age groups, and between genders. ${ }^{[20]}$ The results showed that except for gender differences, the other hypotheses were held true. Female staff nurses scored significantly lower; that is, they favored the traditional model of governance, while males scored higher in most of the subscales except the Resource and Control subscales. Other researchers studied the relationship between Hess's IPNG sores and nursing education, work experience, national certi- fication, employment position, type of work setting (inpatient vs. ambulatory), participation in SG, and age. ${ }^{[21]}$ In contrast to the Lamoureux et al. (2014) ${ }^{[20]}$ study, the results showed no significant relationships among demographic measures and IPNG scores. ${ }^{[21]}$ However, nurses who had a role in SG and worked in the inpatient setting reported higher IPNG scores than ambulatory care nurses. The authors concluded that this may have been because ambulatory care nurses may have had a more difficult time arranging coverage for their patients to enable them participate in SG activities. The authors also added that facilitating engagement of nurses for participation in SG councils may be difficult because of coverage issues. They recommended that management should provide staffing support and praise nurses for serving on UBCs for the SG model to function effectively.

In a longitudinal, non-experimental study in a large medical center in Southeastern United States, Frith and Montgomery (2006) used a survey and focus group to compare SG perception among clinical staff at two points in time: pre-implementation of SG, and, one-year post implementation; and to determine areas for improvement in the SG structure and processes. ${ }^{[22]}$ The survey results showed that more than half of the participants perceived the following SG goals as improved: "communication, accomplishment of unit and nursing goals, decision making in patient care, and educational opportunities" (p. 277). However, turnover and retention were rated poorly. As well, regarding the goal of staff retention, staff who had not participated in SG activities rated it as being worse, while those who had participated rated it as being worse. Staff had similar perceptions of staff turnover with $22 \%$ of staff who had not participated in SG rating it as being worse, but $9 \%$ of those who had been involved in SG rating it as being worse. Therefore, participation in SG activities had an influence on nurses' perceptions of retention and turnover. The overall results of the study showed a decrease in perception and knowledge of SG between the two survey periods. The authors attributed this to the "idealism of shared governance in the pre-implementation period compared to the reality of implementing shared governance" (p. 273).

In the focus groups in the study by Frith et al. (2006), participants shared that some UBCs were slow to start and were still clarifying their roles as well as the respective roles of unit managers, unit council chairs and council members. ${ }^{[22]}$ Council chairs, as well, had to establish their level of authority, develop leadership skills, and learn how to use the organization's resources. Moreover, participants stressed the importance of communicating UBC activities of non-council members. Most importantly, this study showed that staff nurses were aware that SG was "a process, not a project, and 
that it takes time to share responsibility, accountability, and authority for nursing practice" (p. 273).

Based on the literature review, the following four major recurring themes were identified: leadership implications, increased job satisfaction, improved patient care, and improve work environment. Table 1 outlines these themes and their sub-themes.

Table 1. Grouping of findings into themes and subthemes

\begin{tabular}{|c|c|c|c|}
\hline $\begin{array}{l}\text { Theme 1: Leadership and } \\
\text { management }\end{array}$ & $\begin{array}{l}\text { Theme 2: Increased job } \\
\text { satisfaction }\end{array}$ & $\begin{array}{l}\text { Theme 3: Improved patient } \\
\text { care }\end{array}$ & $\begin{array}{l}\text { Theme 4: Improved work } \\
\text { environment }\end{array}$ \\
\hline $\begin{array}{l}\text { Sub-themes } \\
\text { - Supportive and flexible } \\
\text { management } \\
\text { - Effective organizational structure } \\
\text { and support } \\
\text { - Accountability, collaboration, } \\
\text { team work, and professional } \\
\text { responsibilities } \\
\text { Empowerment }\end{array}$ & $\begin{array}{l}\text { Sub-themes } \\
\bullet \text { Engaged in decision } \\
\text { making } \\
\bullet \text { Engaged in SG activities }\end{array}$ & $\begin{array}{l}\text { Sub-themes } \\
\text { - Structural empowerment } \\
\text { - Engaged in decision } \\
\text { making } \\
\text { - Engaged in SG activities } \\
\text { - High quality of care } \\
\text { - Nurses having control over } \\
\text { their practice (managing } \\
\text { patient care) } \\
\text { - Patient-centered culture }\end{array}$ & $\begin{array}{l}\text { Sub-themes } \\
\text { - Nurses having control over } \\
\text { their work environment } \\
\text { - SG contributing to } \\
\text { environment improvement }\end{array}$ \\
\hline
\end{tabular}

\subsection{Leadership and management}

There are leadership implications to nursing leaders for the success of SG and UBC models of governance. As SG is a journey, nursing leaders should give ongoing support to nurses. ${ }^{[14,16]}$ Nursing leaders should give nurses appropriate levels of decisional powers to make decisions about their work environment and nursing practice at the point of care. ${ }^{[14,23]}$ Nursing leaders would benefit from the use of valid and reliable tools to give them insight into the perception of SG model at both the organizational and unit levels. $^{[14]}$

To better support nurses in their autonomous professional nursing practice it is necessary to reorganize the organization's structures and processes. ${ }^{[17]}$ Other organizational implications include supporting nurses in their participation of SG activities by ensuring patient care coverage and compensating them for their commitment. ${ }^{[19]}$ As well, others who explored the lived experience of nurse managers and staff nurses in SG reported that nurse managers themselves believed that mentoring staff was crucial in successful SG implementation, and recognized the importance of letting go of traditional way of management and act as facilitators in the SG process. ${ }^{[23]}$ Nursing leaders should seek out partnerships with staff nurses to create a foundation for a successful SG journey. ${ }^{[23]}$ Other factors that support the successful implementation of SG included organizational structures and leadership practices necessary for creating healthy work environments that promote nurses having control over their nursing practice. ${ }^{[24]}$ Other managerial qualities that are needed to help the transition into an SG model of governance include flexibility, critical listening, and willingness to trust

Published by Sciedu Press and support the transition team. ${ }^{[24]}$

\subsection{Increased job satisfaction}

The autonomous participation in decision-making processes contributes to frontline staff to achieve job satisfaction. ${ }^{[25-27]}$ Engagement in decision-making offered nurses a sense of having control over their practice and having a voice in their patients' care planning. Further, the autonomy nurses had in their work and the extent of their interactions with other nurses and physicians have also been reported to play a significant role in their job satisfaction. ${ }^{[27]}$ Lastly, the total years of experience working in their current units had also a significant relationship with their level of job satisfaction. Nurses who had worked between 16 to 20 years in their current unit had the highest level of satisfaction, while nurses with 10 to 15 years of experience in their current unit had the lowest level. Educational background was another factor. Nurses with diploma degrees were more satisfied about their job compared to those with bachelor degrees. ${ }^{[27]}$

\subsection{Improved patient care}

High levels of nurse engagement in SG activities such as participating in hospital policy decisions affecting nurses' daily work has been associated with quality and safety outcomes. ${ }^{[26]}$ The higher the engagement, the higher the quality and safety outcomes. Staff nurses hold vital knowledge of their patients' needs and can easily identify enablers in the care delivery process. Such knowledge is critical for promoting safe, quality care that meets patient needs. As engagement in SG activities empowers nurses to have control over their practice, they leverage such knowledge to affect change in patient quality and safety. Rather than merely rely- 
ing on nursing management to solve patient care problems, nurses need to develop their own management knowledge and skills. ${ }^{[17]}$

\subsection{Improved work environment}

Shared governance is linked to improved work environments for nurses. Healthy environments result from true partnerships and collaboration among nurses, managers, and educators. ${ }^{[25]}$ Therefore, SG serves as a potential model for promoting an interdisciplinary collaboration and creating an "organizational culture that develops innovation and ensures better care and safety for patients" (p. 1419).

There are benefits and barriers to SG and how SG is used to empower nurses. ${ }^{[28]}$ Nurses working in organizations with SG structures become managers of their patients' care, or bedside leaders. That is because such structures can give nurses a greater autonomy in practice, which therefore enhances their work environment and attracts nurses who are looking for professional and personal career development. Also, SG is a structure that depends on the empowerment of healthcare professionals who are closest to the patient. Shared governance gives nurses their sense of worth and feeds nurses with passion and a need to improve the environment they work in, not only for themselves, but also for their patients.

\section{Discussion}

This literature review was undertaken in order to explore the lived experience of nurses serving on UBCs and how they perceived SG. The retrieved articles highlighted the lived experiences of nurses with UBCs and SG models of governance as well as some key factors for the success and effectiveness of these models.

Nurses' perceptions of successful SG were found to vary according to several factors. Supportive leadership and management and effective organizational structure have been identified as one of these factors necessary for the success of SG. ${ }^{[14,16,17,19,22-24]}$ These factors are important because they promote partnership, team effort, and collaboration among nurses and between nurses and their nurse managers. These were needed in successfully transitioning from a traditional to an SG structure. Other enablers for SG success include the accountability of council members, and an effective council chair who is organized, dependable, and has effective communication skills.

Job satisfaction is the most frequently used measure in nursing literature and has a strong influence on many aspects of nursing. ${ }^{[29]}$ Several factors have been associated with nurses' job satisfaction. It influences nurses' perceptions and sup- port of SG. ${ }^{[25-27]}$ A major component of job satisfaction that mainly concerns SG is the degree of nurses' participation in point of care decisions. Staff who perceive an opportunity to speak up for their issues and concerns and be involved in decision-making about issues affecting their work will be more likely to express satisfaction at work. ${ }^{[2]}$ Hospitals are increasingly focusing on enhancing staff satisfaction to increase their retention through the promotion of a healthy work environment and professional development. ${ }^{[30]}$ Hospitals that have successfully achieved Magnet designation are known for fostering positive work environments for their employees. ${ }^{[30]}$ Nurses' age, years of education and their educational background have also been shown to be related to their job satisfaction. Older nurses and total years of experience in their current working unit are more satisfied and engaged in SG activities. Baccalaureate prepared nurses are less satisfied with their engagement in SG activities then Diploma prepared nurses. ${ }^{[27]}$

The concept of structural empowerment in nursing has been associated with quality of care, and hospitals that provide nurses with opportunities to be engaged in SG are more likely to provide better patient experiences and better quality of care. ${ }^{[26]}$ Nursing governance is about managing care, and the main responsibility of clinical nurses is to manage patient care as they are in a central position in a healthcare organization and in the provision of patient care. ${ }^{[17]}$ This is important because nursing governance is centered on managing patient care quality, and its goal is establishing and maintaining standards of care excellence. Structural empowerment for nurses' work life experience is important. ${ }^{[31]}$ Empowerment creates positive nursing professional practice environments that increase their satisfaction with their work the quality of care they provide. ${ }^{[31]}$

\section{SUMMARY AND CONCLUSION}

Staff nurses serving on UBCs at HMC have different perspectives on the success of the implementation of SG and UBCs. Staff nurses are of the view that the introduction of such a governance model has not made any difference. Further, they are confused about what is expected of them and what their exact role is serving on councils. The evidence suggests that $\mathrm{SG}$ is a journey that requires continuous support and assessment from nurse leaders for any new issues that may arise and challenges that may arise at any stage of its implementation. Models of SG can struggle at any step of implementation, and need organizational support to move forward. Job satisfaction can greatly influence nurses' perceptions and support of UBCs and SG, and staff who perceive an opportunity to speak up for their issues and concerns and be involved in decision making about their work will be 
more likely to express satisfaction at work. Nurses' commitment to participate in SG activities depends on factors such as nursing education, years of experience, employment status, and experience with councils. Structural empowerment in nursing is crucial, and hospitals that provide nurses with opportunities to be engaged in SG are more likely to provide better patient experiences and better quality of care.

\subsection{Implications}

As SG is characterized as a journey, it is necessary for the nursing leadership to monitor its implementation on an ongoing basis. The success of SG needs collaborative leadership engagement with staff nurses to empower them to take ownership of UBCs and SG. Nursing leadership can increase understanding and knowledge of these structural and process models by supporting UBCs maintaining an open communication between nursing administration and staff nurses.

\subsection{Recommendations}

Based on the synthesis of the relevant literature on the lived experience of nurses serving on UBCs and their experience with SG and the scarcity of research on this subject in Qatar supports a primary research study in the future. This literature review has the potential to inform the design and conduct of a qualitative study for the benefit of informing improvements in SG and UBC models implementation.

\section{Conflicts of InTEREST Disclosure}

The authors declare that there is no conflict of interest.

\section{REFERENCES}

[1] Van Bogaert PV, Peremans L, Diltour N, et al. Staff Nurses' Perceptions and Experiences about Structural Empowerment: A Qualitative Phenomenological Study. PLOS ONE. 2016; 11(4): 1-14.

[2] Barden AM, Griffin MTQ, Donahue M, et al. Shared Governance and Empowerment in Registered Nurses Working in a Hospital Setting. Nursing Administration Quarterly. 2011; 35(3): 212-218. PMid:21654480 https : //doi.org/10.1097/NAQ.0b013e3181 ff 3845

[3] Bogue RJ, Joseph ML, Sieloff CL. Shared governance as vertical alignment of nursing group power and nurse practice council effectiveness. Journal of Nursing Management. 2009; 17: 4-14. PMid:19166517 https://doi.org/10.1111/j.1365-2834.20 $08.00954 . x$

[4] Kramer M, Schmalenberg C, Maguire P, et al. Walk the talk: promoting control of nursing practice and a patient-centered culture. Critical Care Nurse. 2009; 29(3): 77-93. PMid:19487783 https: //doi.org/10.4037/ccn2009586

[5] Laschinger HKS, Nosko A, Wilk P, et al. Effects of unit empowerment and perceived support for professional nursing practice on unit effectiveness and individual nurse well-being: A time-lagged study. International Journal of Nursing Studies. 2014; 51: 1615-1623. PMid:24810929 https://doi.org/10.1016/j.ijnurstu. 201 4.04 .010

[6] Laschinger HKS, Wilk P, Cho J, et al. Empowerment, engagement and perceived effectiveness in nursing work environments: does experience matter? Journal of Nursing Management. 2009; 17: 636-646. PMid:19575722 https://doi.org/10.1111/j.1365-2834.20 08.00907. $\mathrm{x}$

[7] Wang S, Liu Y. Impact of professional nursing practice environment and psychological empowerment on nurses' work engagement: test of structural equation modelling. Journal of Nursing Management. 2015; 23: 287-296. PMid:24112142 https://doi.org/10.1111/ jonm. 12124

[8] Wagner JIJ, Warren S, Cummings G, et al. Canadian Journal of Nursing Research. 2013; 45(4): 108-128.

[9] Anthony M. Shared Governance Models: The Theory, Practice, and Evidence. Online Journal of Issues in Nursing. 2004; 9(1): 13-13. PMid:14998353

Published by Sciedu Press
[10] Fray B. Evaluating Shared Governance: Measuring Functionality of Unit Practice Councils at the Point of Care. Creative Nursing. 2011; 17(2): 87-95. https://doi.org/10.1891/1078-4535.17.2.8 7

[11] Hamad Medical Corporation. (n.d). Our Organization. Available from: https://www.hamad.qa/EN/About-Us/Our-Organizat ion/Pages/default.aspx

[12] American Nurses Credentialing Center. Overview of ANCC magnet recognition program new model. 2011. Available from: http://nursecredentialing.org/Documents/Magne t/MagOverview-92011.pdf

[13] LoBiondo-wood G, Haber J. Nursing research in Canada (3ed) Toronto, Canada: Elsevier Canada. 2013.

[14] Gerard SO, Owens DL, Oliver P. Nurses' Perception of Shared Decision-Making Processes Quantifying a Shared Governance Culture. Journal of Nursing Administration. 2016; 46(9): 477-483. PMid:27556657 https://doi.org/10.1097/NNA . 0000000000 000378

[15] Mangold KL, Pearson KK, Schmitz JR, et al. Perceptions and characteristics of registered nurses' involvement in decision making. Nursing Administration Quarterly. 2006; 30(3): 266-272. https: //doi.org/10.1097/00006216-200607000-00011

[16] Winslow S, Hougan A, DeGuzman P, et al. What's being said about shared governance? Nursing Management. 2015; 46(4): 46-51. PMid:25798770 https://doi .org/10.1097/01. NUMA . 000046 2366.91153.e2

[17] dos Santos JLG, Erdmann AL. Governance of professional nursing practice in a hospital setting: a mixed methods study. Rev. Latino-Am. Enfermagem. 2015; 23(6): 1024-1032. PMid:26625992 https://doi.org/10.1590/0104-1169.0482.2645

[18] Al-Faouri IG, Al Ali N, Essa MB. Perception of Shared Governance among Registered Nurses in a Jordanian University Hospital. International Journal of Humanities and Social Science. 2014; 4(1): 254-262.

[19] Wilson J, Speroni KG, Jones RA, et al. Exploring how nurses and managers perceive shared governance. Research Corner. 2014; 44(7): 19-22.

[20] Lamoureux J, Judkins-Cohn T, Butao R, et al. Measuring perceptions of shared governance in clinical practice: psychometric testing of the 
RN-focused Index of Professional Governance (IPNG). Journal of Research in Nursing. 2014; 19(1): 69-87. https ://doi.org/10.1 $177 / 1744987113504409$

[21] Overcash J, Petty LJ, Brown S. Perceptions of shared governance among nurses at a Midwestern hospital. Nursing Administration Quarterly. 2012; 36(4): E1-E11. PMid:22955226 https ://doi.or g/10.1097/NAQ.0b013e318268961b

[22] Frith K, Montgomery M. Perceptions, knowledge, and commitment of clinical staff to shared governance. Nursing Administration Quarterly. 2006; 30(3): 273-284. https ://doi.org/10.1097/000062 16-200607000-00012

[23] Ott J, Ross C. The journey toward shared governance: the lived experience of nurse managers and staff nurses. Journal of Nursing Management. 2014; 22: 761-768. PMid:23441926 https: //doi.org/10.1111/jonm.12032

[24] Dunbar B, Park B, Berger-Wesley M, et al. Shared governance making the transition in practice and perception. Journal of Nursing Administration. 2007; 37(4): 177-183.

[25] dos Santos JLG, Erdmann AL, Andrade SR, et al. Nursing governance: an integrative review of the literature. Revista da Escola de Enfermagem da USP. 2013; 47(6): 1414-21.
[26] Kutney-Lee A, Germack H, Hatfield L, et al. Nurse Engagement in Shared Governance and Patient and Nurse Outcomes. Journal of Nursing Administration. 2016; 46(11): 605-612. PMid:27755212 https://doi.org/10.1097/NNA.0000000000000412

[27] Shwaihet NH, Nasaif HA. The relationship of participation in shared governance to work satisfaction among cardiovascular nurses working in a tertiary hospital in Saudi Arabia. Clinical Nursing Studies. 2015; 3(4).

[28] Taylor K. Using shared governance to empower nurses. Nursing Times. 2016; 112(1/2): 20-23.

[29] O’May F, Buchan J. Shared governance: a literature review. International Journal of Nursing Studies. 1999; 36: 281-300. https : //doi.org/10.1016/S0020-7489(99)00023-1

[30] Fisher CA, Jabara J, Poudrier L, et al. Shared governance: the way to staff satisfaction and retention. Nursing Management (Springhouse). 2016; 47(11): 14-16. PMid:27787399 https ://doi .org/10.109 7/01. NUMA. 0000502808.67918.e8

[31] Laschinger HKS. Effect of empowerment on professional practice environments, work satisfaction, and patient care quality further testing the nursing worklife model. Journal of Nursing Care Quality. 2008; 23(4): 322-330. PMid:18431259 https://doi.org/10.1097/01 .NCQ.0000318028.67910.6b 